\title{
A Case with Spondyloepiphyseal Dysplasia Tarda with TRAPPC2 Mutation
}

\author{
Hyun-Jin Kim, Beom-Hee Lee ${ }^{1,2}$, Yoo-Mi Kim, Gu-Hwan Kim², Ok-Hwa Kim³ and Han-Wook Yoo ${ }^{1,2^{*}}$ \\ ${ }^{1}$ Department of Pediatrics, Asan Medical Center, University of Ulsan College of Medicine, Seoul, Korea \\ ${ }^{2}$ Medical Genetics Center, Asan Medical Center, University of Ulsan College of Medicine, Seoul, Korea \\ ${ }^{3}$ Department of Radiology, Ajou University Hospital, Suwon, Korea
}

Spondyloepiphyseal dysplasia tarda (SEDT) is an X-linked skeletal dysplasia. Patients show disproportionate short stature with short trunk and barrel-shaped chest, which usually become pronounced in late childhood. The radiologic findings are characterized by narrow intervertebral disc spaces and moderate epiphyseal dysplasia of long bones. Here we report a case of SEDT with a novel frameshift mutation in TRAPPC2, the disease-causing gene of SEDT. This is the first Korean report with SEDT confirmed by genetic testing.

Key Words: X-linked skeletal dysplasia, TRAPPC2 gene, Spondyloepiphyseal dysplasia tarda

\section{Introduction}

Spondyloepiphyseal dysplasia tarda (SEDT; OMIM 313400) is an X-linked skeletal dysplasia that primarily affects the vertebral bodies and epiphyses. Spondyloepiphyseal dysplasia has been divided into a congenital and a tarda form according to the age of onset and clinical severity." The different modes of inheritance within the group reflect the genetic heterogeneity. Autosomal dominant, autosomal recessive, and $\mathrm{X}$-linked recessive patterns of inheritance have been described. ${ }^{2,3)}$

$X$-linked SEDT is caused by mutations in the Trafficking protein particle complex, subunit 2 (TRAPPC2) gene. This gene contains six exons, spanning about 20kb of genomic DNA in Xp22. ${ }^{4)}$ This disorder is usually identified in late childhood males with disproportionate short stature with short trunk and barrel-shaped chest. Heterozygous female carriers are usually clinically and radiographically normal. ${ }^{5}$ Radiologic evaluations can reveal narrow disc spaces and moderate epiphyseal dysplasia. These characteristic constellation of radiologic findings can be seen in the lateral view of the thoraco-lumbar spine; narrowing of intervertebral disc spaces, and pathognomonic superior and inferior 'humps' involving the posterior two-thirds of the flattened vertebral bodies. ${ }^{6}$

In most cases, there's no specific or mild subjective symptom. However, severe epiphyseal dysplasia can lead to premature secondary osteoarthritis affecting the spine and hip joints, often requiring hip replacement and resulting in significant morbidity. Here we report a case of SEDT with a novel frameshift mutation in TRAPPC2. To our knowledge, this is the first Korean report with SEDT confirmed by genetic testing.

Received: 10 May 2012, Revised: 20 June 2012, Accepted: 22 June 2012, Published: 30 June 2012

${ }^{*}$ Corresponding author: Han-Wook Yoo

Department of Pediatrics, Asan Medical Center, University of Ulsan College of Medicine, 86, Asanbyeongwon-gil, Songpa-gu, Seoul 138-736, Korea Tel: +82-2-3010-3374, Fax: +82-2-473-3725, E-mail: hwyoo@amc.seoul.kr

(c) This is an open-access article distributed under the terms of the Creative Commons Attribution Non-Commercial License (http://creativecommons.org/licenses/by-nc/3.0/) which permits unrestricted non-commercial use, distribution, and reproduction in any medium, provided the original work is properly cited.

(c) Copyright 2012 by the Korean Society of Medical Genetics 


\section{Case Report}

A 15-year-old boy visited our hospital due to disproportionate short stature. His birth weight and height was within normal range without perinatal problems. His developmental milestones were normal. His height was $147.6 \mathrm{~cm}$ (-2.66 SDS). His father's height was $178 \mathrm{~cm}(0.81$ SDS), whereas his mother was proportionately short at $146 \mathrm{~cm}(-3.12$ SDS). His two sisters were $158 \mathrm{~cm}(-0.55$ SDS) and $160 \mathrm{~cm}(-0.15$ SDS) tall (Fig. 1A). His face was not dysmorphic and intelligence was normal. Normal ambulatory activities were possible without help. Trunk was relatively short and chest cage was barrel-shaped. Range of motion was not limited in all of the axial and peripheral joints.
Pain, tenderness or swelling was not noticed. The shapes of his hands and feet were normal.

Skeletal survey revealed mild flattening of the epiphyses of long bones in knee and hip, but the metaphyses and diaphyses were normal. In addition, platyspondyly, reduced intervertebral spaces, bell shaped thorax, and scoliosis were observed. Femoral head was flattened and femoral neck was short and broad. The phalangeal bones of both hands and feet were normal (Fig. 2). With the impression of SED, genetic testing was done for COL2A1, which was normal. Next, we searched TRAPPC2 gene, in which a frameshift mutation was found, c.40del (p.Asp14llefs*24). This mutation was not previously reported. His mother was heterozygous carrier for the mutation, whereas his two sisters didn't harbor the mutation

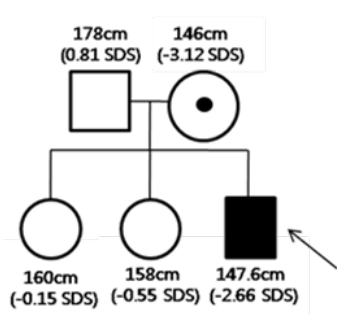

A

Fig. 1. A) Pedigree of a SEDT family. Black box represents an affected male proband. B) Chromatograms of partial DNA sequence of TRAPPC2. The patient harbors c.40del (p.Asp14llefs*24), and his mother was a heterozygote carrier for the frame shift mutation. The deletion site was indicated with arrows.

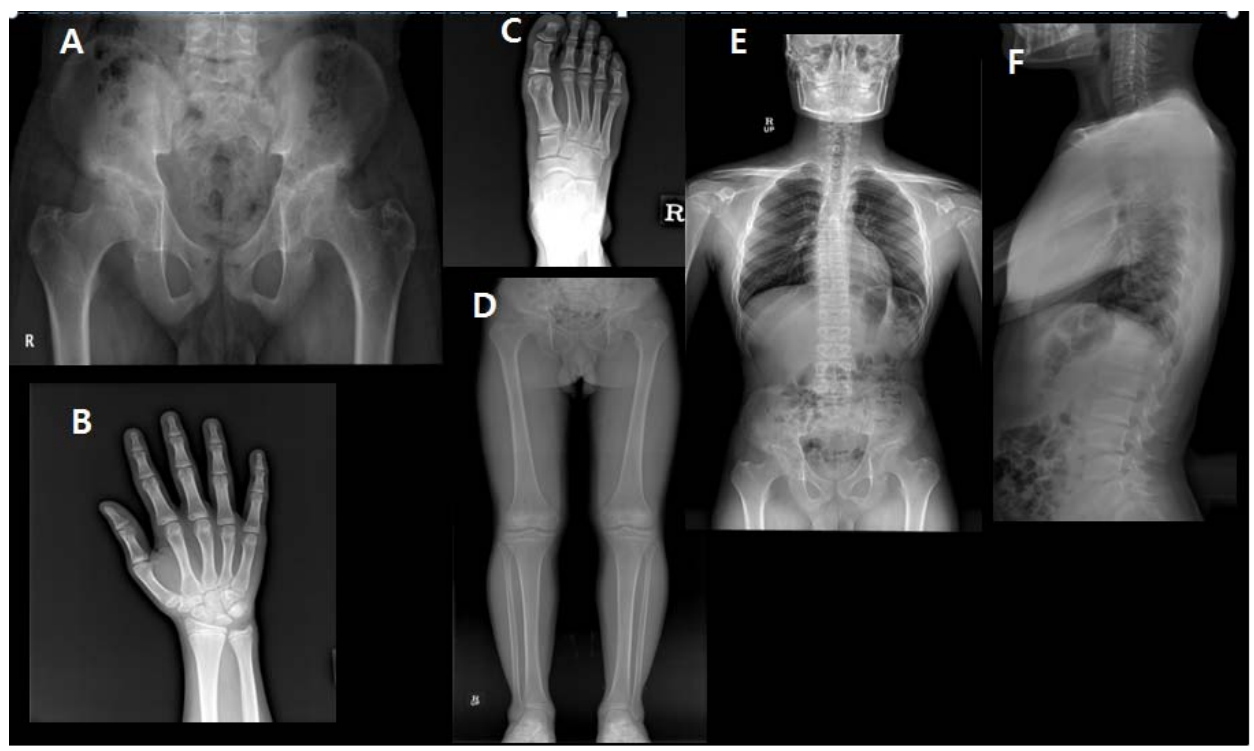

Fig. 2. A) Anteroposterior X-ray of the pelvis shows short and broad femoral neck and flattened femoral heads. Mild flattening of the epiphyses of long bones was also seen. $B-D$ ) There is no definite abnormality in the metaphyses of long bones and phalanges of hands and feet. $E$ - $F$ ) Intervertebral disc spaces were decreased and scoliosis and platyspondyly were noted. 
(Fig. 1B). His mother didn't complain of back or hip pain without a radiologic evidence of spine or joint involvement.

\section{Discussion}

SED was first described by Wynne-Davies in 1982, as an initial series of 15 patients. More detailed clinical and radiological description was done by Spranger et al. in a series of six patients. In autosomal dominant and recessive SEDs, symptoms are noted in childhood and progressive arthropathy is a common presentation. The thoracic spine is most common affected site. Meanwhile, symptoms are usually minimal before 12 years in X-linked recessive SEDT and hip is most frequently involved. ${ }^{2,7}$ Autosomal dominant SED is caused by mutations of type Il collagen gene, $C O L 2 A 1$, and autosomal recessive SED by the ATPSK2 gene. ${ }^{3,8)}$

TRAPPC2 gene contains six exons, spanning about $20 \mathrm{~Kb}$ of genomic DNA in Xp 22. The function of TRAPPC2 protein is not clear. However, it is thought to be a member of large multisubunit complex involved in targeting and fusioning of endoplasmic reticulum to Golgi transport vesicles with their acceptor compartment. Therefore, the loss of the function of TRAPPC2 can cause abnormal cellular transport of cartilage proteins. ${ }^{4}$ To date, about fifty mutations have been reported. Most mutations are frameshift or missense mutations, whereas splicing and gross deletion mutations have been identified in a small proportion of the patients

SEDT progressively leads to deformities of small and large joints and irregularities of end plates of vertebral bodies. Progressive limitations of motion in joints result in gait disturbance. Because the clinical symptom of SEDT is pronounced in late childhood, it is very difficult to give an optimal management before degenerative and restrictive skeletal deformities develop. Many cases can remain undiagnosed until later decades when this condition becomes superimposed by secondary arthritic changes. The major complication of this disorder is premature arthritis predominantly affecting the spine and hip joints. ${ }^{9)}$ It can be misdiagnosed as juvenile idiopathic arthritis (JIA) due to the similarity of clinical manifestations. ${ }^{6,10)}$ Both are characterized by abnormal shape and structural deformities of the long bones, hands, and feet. Family histories, typical radiologic finding, normal laboratory investigations (normal acute phase reactants such as C-reactive protein, erythrocyte sedimentation rate and rheumatoid factor), absence of synovial inflammation in soft tissue swelling and effusion can help make a diagnosis of SEDT.

The treatment for SEDT issupportive. Rehabilitation and surgical procedures may provide symptomatic relief. Some patients may need medications including non-steroidal anti-inflammatory medications or opiates. Efficacy of growth hormone therapy on final adult height has not been reported in this condition. Lack of response to anti-rheumatic medications is another finding differentiating SEDT from JIA.

Only a few cases of SED have been described in Korea. One case was diagnosed as SED congenita with COL2A1 mutations.11) The other case was misdiagnosed as having ankylosing spondylitis and treated with antirheumatic drug in ten years. After radiographic evaluations, SEDT was diagnosed clinically, but without genetic testing. ${ }^{12)}$

Most SEDT patients are expected to have normal life, although the joint deformities are progressive and degenerative or restrictive changes can lead to severe joint impairments. Short stature is common in this condition and ongoing psychosocial support of the patient and the family is important with appropriate genetic counseling.

\section{Acknowledgement}

We thank our patientand hisfamily members for their participation in this study. This study was supported by Grant A080588 from the Korean Ministry of Health, Welfare and Family Affairs.

\section{References}

1. Anderson IJ, Goldberq RB, Marion RW, Upholt WB, Tsipouras P. Spondyloepiphyseal dysplasia congenita: genetic linkage to type II collagen (COL2AI). Am J Hum Genet 1990;46:896-901.

2. Kaptanoqlu E, Percin F, Percin S, Torel-Ergur A. Spondyloepiphyseal dysplasia tarda with progressive arthropathy. Turk J Pediatr 2004;46: 380-3.

3. Leroy JG, Leroy BP, Emmery LV, Messiaen L, Spranger JW. A new type of autosomal recessive spondyloepiphysela dysplasia tarda. Am J Med Genet A 2004;125:49-56.

4. Guo H, Xu X, Wang K, Zhang B, Deng G, Wang Y, Bai Y. A novel RNAsplicing mutation in TRAPPC2 gene causing $X$-linked Spondyloepiphyseal dysplasia tarda in a large Chinese family. J Genet 2009;88:87-91.

5. Mumm S, Christie PT, Finnegan P, Jones J, Dixon PH, Pannett AA, Harding B, et al. A Five-Base Pair Deletion in the Sedlin Gene Causes Spondyloepiphyseal dysplasia tarda in a six-generation Arkansas kindred. J Clin Endocrinol Metab 2000;85:3343-7.

6. Bal S, Kocyiqit H, Turan Y, Gurgan A,Bayram KB, Guvenc A, Kocaaga Z, et al. Spondyloepiphyseal dysplasia tarda ;four cases from two familes. Rheumatol Int 2009;29:699-702. 
7. Iceton JA, Horne G. Spondyloepiphyseal dysplasia tarda. The X-linked variety in three brothers. J Bone Joint Surg Br 1986;68:616-9.

8. Faiyaz ul Haque M, King LM, Krakow D, Cantor RM, Rusiniak ME, Swank RT, et al. Mutations in orthologous genes in human spondyloepimetaphyseal dysplasia and the brachymorphic mouse. Nat Genet 1998;20: 157-62.

9. Savarirayan R, Thompson E, Gecz J. Spondyloepiphyseal dysplasia tarda (SEDL,MIM \#313400) . Eur J Hum Genet 2003;11:639-42.
10. Spranger J. Albert C, Schilling F, Bartsocas C, Stoss H. Progressive pseudorheumatoid arthritis of childhood(PPAC).A hereditary disorder simulating rheumatoid arthritis. Eur J Pediatr 1983;40:34-40 .

11 Jung SC, Mathew S, Li OW, Lee YJ, Lee KS, Song HR. Spondyloepiphyseal dysplasia congenital with absent femoral head. J Pediatr Orthop B 2004;13:63-9.

12 Oh IH, Song JS, Rim DH, Choi JW, Lee SH, Lee JH, Kim TH. A case of spondyloepiphyseal dysplasia tarda misdiagnosed as ankylosing spondylitis. J Rheumatol 2011;18:311-4. 